\title{
Diacronie
}

Studi di Storia Contemporanea

$N^{\circ} 26,2 \mid 2016$

Un bilancio della scommessa democratica della Chiesa cattolica

\begin{abstract}
Alexander Höbel, Luigi Longo, una vita partigiana (1900-1945)
\end{abstract}

\section{Giulia Strippoli}

\section{(2) OpenEdition \\ Journals}

Edizione digitale

URL: http://journals.openedition.org/diacronie/4026

DOI: $10.4000 /$ diacronie.4026

ISSN: 2038-0925

\section{Editore}

Association culturelle Diacronie

Notizia bibliografica digitale

Giulia Strippoli, « Alexander Höbel, Luigi Longo, una vita partigiana (1900-1945) », Diacronie [Online], N

26,2 | 2016, documento 10, Messo online il 29 juin 2016, consultato il 24 septembre 2020. URL

http://journals.openedition.org/diacronie/4026 ; DOI : https://doi.org/10.4000/diacronie.4026 


\section{Diacronie}

N. 26 | 2|2016 Un bilancio della scommessa democratica della Chiesa cattolica

10/

\section{RECENSIONE:}

\section{Alexander HÖBEL, Luigi Longo, una vita partigiana (19oo-1945), Roma, Carocci, 2013, 374 pp.}

di Giulia STRIPPOLI *

Luigi Longo (Fubine, 15 marzo 1900 - Roma, 16 ottobre 1980) fu un dirigente comunista politicamente abile, strategicamente intelligente, coraggioso, leale, disciplinato. Fu guida e capo nonostante la scelta di essere il secondo di Togliatti. La freddezza, la calma e l'imperturbabilità celavano una dimensione tutta umana in cui risaltano sensibilità e generosità verso i compagni, il partito, la famiglia. Il libro di Alexander Höbel ricostruisce con chiarezza e approfondimento il percorso politico di "Gallo" (questo era il suo nome di battaglia) dalla nascita nel Monferrato fino alla liberazione dal nazifascismo, attraversando la formazione del Pcd'I, la crisi dello stato liberale, l'avvento del fascismo, la guerra di Spagna e la Seconda guerra mondiale. L'autore ha scelto un'impostazione classica per colmare una lacuna storiografica sulla vita del dirigente comunista. Il libro inizia con la definizione dell'ambiente famigliare e il richiamo alle condizioni di vita nella campagna piemontese del primo Novecento. La prima immagine che abbiamo di Longo è quella di un bambino cresciuto in condizioni economiche difficili, trasferitosi presto a Torino con la famiglia, dove, dalla mescita di vino gestita dai genitori, venne in contatto con l'ambiente operaio della città, fatto di miseria, lotte e solidarietà. L'immagine che chiude il libro è invece una fotografia del 6 maggio 1945, in cui Longo è ritratto insieme ad altri componenti del CVL. Longo è ormai l'eroe della Resistenza, vicesegretario del partito insieme a Secchia, in piena sintonia con Togliatti, ma con idee politiche proprie. L'immagine dell'abbraccio alla moglie Teresa Noce ritrovata dopo le drammatiche vicende della guerra e della deportazione suggella l'epopea di un uomo indagata con un amplissimo uso delle fonti 
d'archivio che l'autore usa per far risaltare un percorso drammatico ma luminoso, dove le ombre e le critiche, quando ci sono, rimangono irrilevanti.

L'inizio e la fine del libro sono un buon esempio delle due criticità principali di questa biografia, che è sostanzialmente una storia del Pcd'I - dagli anni della fondazione alla liberazione - dal punto di vista di Longo. Le prime due pagine tracciano un profilo definito in partenza usando le parole dello stesso Longo: il carattere riservato perché il molto parlare delle sorelle lo obbligava a tacere ${ }^{1}$, l'abbandono della campagna, dove si era sentito partecipe dell'umiliazione della povera gente, condividendone i sentimenti di rancore e offesa ${ }^{2}$, e l'incontro con l'«eroismo vero e proprio»3 degli operai che partecipavano alle lotte torinesi. La memoria di Longo apre bene la biografia, immerge il lettore nel primo Novecento della campagna monferrina e nell'atmosfera di fatica e repressione di Barriera di Milano, ma uno sguardo critico dell'autore sul ruolo della memoria del protagonista nel raccontare la propria formazione avrebbe forse fornito qualche spunto per riflettere non solo sulla traiettoria di Longo e del Pcd'I, ma anche sulla storiografia riguardante i dirigenti comunisti e il partito. La seconda criticità si riferisce alla dimensione dell'epopea, con molte luci e rarissime ombre, che caratterizza il libro e che nella conclusione emerge in maniera palese. Pur riconoscendo la statura eccezionale di "Gallo", il lettore rimane perplesso dalla conferma da parte dell'autore dell'esemplarità della vita di Longo, «non solo nella storia dell'Italia democratica ma anche di tutto il movimento operaio internazionale», e del carattere partigiano della vita dettato da un sentimento di appartenenza di classe che mai (quel mai che richiama una sfera tutta eroica e poco affine alle vicende umane) fu inficiato «da visioni ristrette degli obiettivi di quella che per Longo era la "classe generale", la classe operaia e i lavoratori salariati»4. Il riferimento all'affettuosità della lettera scritta alla Noce e dell'abbraccio tra i due sembra poi cancellare tutto d'un tratto le pur esistenti spie della difficoltà di un rapporto a cui l'autore fa cenno nel corso del libro 5 . L'annullamento del matrimonio e le sue conseguenze ${ }^{6}$ sono successive al 1945, anno in cui termina la narrazione, ma il "lieto fine" scelto dall'autore - che non accenna

${ }^{1}$ HÖBEL, Alexander, Luigi Longo. Una vita partigiana (19oo-1945), Roma, Carocci, 2013, p. 21.

${ }^{2}$ L'autore si basa sulle memorie contenute in: LONGO, Luigi, SALINARI, Carlo, Tra reazione e rivoluzione. Ricordi e riflessioni sui primi anni di vita del PCI, Milano, Edizioni del Calendario, 1972.

3 HÖBEL, Alexander, cit., p. 22.

4 Ibidem, p. 365.

5 Ibidem, p. 273.

${ }^{6}$ Teresa Noce racconta la sofferenza provocata dalla notizia della contraffazione della sua firma per ottenere l'annullamento del matrimonio e di come la sua scelta di rivolgersi alla commissione centrale di controllo del partito per denunciare la vicenda abbia portato alla sua esclusione dalla direzione. Cfr. NOCE, Teresa, Rivoluzionaria professionale, Milano, La Pietra, 1974, pp. 408-411. 
nemmeno in nota ai successivi sviluppi della relazione tra i due - dà l'impressione di un'omissione e conferma che l'autobiografia di Teresa Noce, ampliamente citata, è usata solo per valorizzare la figura di Longo, tralasciando il percorso politico e personale di "Estella". Da un lato, insomma, Longo risulta poco umano per la dimensione di eroismo e di esemplarità, dall'altro l'autore non può dimenticare che è uomo e allora lo ritrae parzialmente come marito (che non sbaglia, al massimo dimostra freddezza) e come padre che prende tra le braccia il primo figlio Luigi Libero in carcere e lo stringe finché le guardie lo permettono ${ }^{7}$ e che insieme a Togliatti fa il baby-sitter a Mosca mentre Rita Montagnana e Teresa Noce lavorano. In questo caso l'autore apre uno spiraglio che forse era dovuto ma che resta circoscritto a questa frase e dunque inefficace, sul rapporto pubblico-privato:

È un'immagine inconsueta di un Longo e un Togliatti familiari, impegnati in quel difficile compito di contemperare il privato e il politico che sarà al centro della vita e delle discussioni di molte generazioni di militanti ${ }^{8}$.

Ritroviamo Longo padre durante la guerra di Spagna quando l'autore parla dello sforzo bellico che non impedisce a "Gallo" né di occuparsi del lavoro politico né di ottemperare ai suoi «doveri di padre» e che scrive a Mosca preoccupato per il figlio Gigi che da quattro anni si trovava presso la casa dei bambini del Soccorso Rosso internazionale e che i coniugi Longo decidono di far rientrare a vivere con loro9. Gli accenni alle vicende famigliari sono sempre accompagnati da una patina di legittimazione, come a sottolineare la rettitudine dell'uomo politico, del marito, del padre.

In rarissime occasioni sembrano presenti degli spunti critici. Il primo riguarda il 1928, quando Longo era dirigente della FGCI e polemizzava con Togliatti sulla parola d'ordine dell'assemblea repubblicana, sbagliando, dice l'autore, a sopravvalutare la radicalizzazione in atto, a contrapporre schematicamente rivoluzione e controrivoluzione, mentre si sarebbe rivelata più adeguata la visione di Togliatti delle soluzioni intermedie ${ }^{10}$. Del resto, poche pagine prima, l'autore aveva riferito che Longo stesso, nelle sue memorie, aveva sì ribadito la critica intorno all'assemblea repubblicana ma aveva riconosciuto la ragione di Togliatti nel criticare le fughe in avanti dei giovani ${ }^{11}$. Poco oltre ${ }^{12}$, troviamo una critica dello schematismo di Longo

7 HÖBEL, Alexander, op. cit., p. 60.

8 Ibidem, p. 76.

9 Ibidem, p. 267.

${ }^{10}$ Ibidem, pp. 105-106.

${ }^{11}$ Ibidem, p. 101. 
corretto da Togliatti nei momenti di preparazione della "svolta" che vedranno l'isolamento di "Gallo", la polemica con Togliatti (senza mai arrivare, però, allo scontro aperto) e il superamento del contrasto grazie all'intento unitario di entrambi ${ }^{13}$. Più che di veri e propri rilievi critici si tratta di ripensamenti delle posizioni di Longo alla luce di quello che sarebbe successo in seguito e usando come contrappunto la prospettiva di Togliatti, con il quale però, si spiega dopo, "Gallo" avrebbe trovato una perfetta sintonia politica.

L'impressione generale che ne deriva è di un percorso definito in partenza dove le critiche sembrano più autocritiche o allineamento a quella che si sarebbe confermata come linea guida del partito Si intende sottolineare, insomma, che la biografia rimane abbastanza schiacciata sul punto di vista di Longo. Le posizioni degli oppositori, suoi o del Pcd'I, non sono approfondite e non ricevono attenzione critica, né dovuto spazio viene lasciato ad alcuni punti cruciali della storia degli inizi del partito come gli allontanamenti prima di Bordiga, poi di Tasca e di Ravazzoli, Tresso, Leonetti e Silone. La voce di costoro, così come la bibliografia esistente intorno a tali temi, è piuttosto smorzata se si considera l'approfondimento con cui l'autore, in altre occasioni, ricostruisce il dibattito interno al partito. Questa sensazione è amplificata dal fatto che in diversi passaggi mancano i riferimenti agli anni in cui avvenivano riunioni, plenum, congressi, spostamenti perché è appunto una scrittura molto "interna". La condivisione e poi il distacco dalle tesi politiche di Bordiga, le istanze gramsciane, la ricerca della sintonia politica con Togliatti preservando sempre l'unità, la chiara presa di posizione a favore di Stalin nel confronto interno al partito bolscevico, sono tappe scandite da una sorta di linearità narrativa che appare un po' eccessiva. E che è enfatizzata dal gusto dell'autore nel rintracciare nel passato di Longo i segnali di intuizioni e "prefigurazioni" di quanto sarebbe avvenuto in futuro. In particolare, Longo avrebbe prefigurato chiaramente la Resistenza, mentre era dirigente del centro estero della FGCI e quasi protagonista della svolta di fine anni Venti, e, successivamente, verso la fine dell' l'esperienza spagnola delle Brigate internazionali. Nel primo caso, Gallo avrebbe anticipato l'idea della Resistenza parlando della lotta armata operaia e contadina contro il fascismo e del suo futuro allargamento ${ }^{14}$. Nel secondo caso, il comandante delle

\footnotetext{
12 Ibidem, p. 111.

13 Anche in un altro momento, troviamo Longo qualificato per lo "schematismo": siamo alla vigilia della politica dei fronti popolari e i comunisti discutono sull'unità di azione con i socialisti; Höbel giudica la polemica antiriformista di Longo nei confronti dei socialisti «sempre molto viva e non priva di schematismi». Cfr. Ibidem, pp. 206-207.

14 Ibidem, p. 106. Anche le osservazioni di Longo e la risoluzione del presidium del Comitern del 1927 vengono categorizzate come prefigurazione: «viene prefigurato, con quasi vent'anni d'anticipo, lo sviluppo della lotta di Liberazione del 1943-45: un movimento popolare armato, di cui le classi lavoratrici sono l'elemento centrale attorno al quale si aggregano altri strati della
} 
Brigate internazionali avrebbe nettamente prefigurato la Resistenza riferendosi al ruolo dei volontari e alla lezione di unità appresa in Spagna15. Il "Gallo" a capo delle "Garibaldi" durante la Resistenza avrebbe invece prefigurato il 25 aprile allorché stabiliva un nesso tra la lotta armata e lo sciopero generale e politico ${ }^{16}$.

Höbel ha scritto una biografia ricca di spunti per il dibattito storiografico e politico intorno a questioni importanti che riguardano sia le narrazioni sui partiti comunisti e i dirigenti comunisti, sia la storia del Pcd'I, del fascismo, della Resistenza. Si capisce fin dalle prime righe che l'autore ha fatto una scelta interpretativa e le criticità rilevate sulla stessa linea se ne potrebbero rilevare altre - derivano da una precisa lettura dell'esperienza di Longo come dirigente del Pcd'I e all'interno del movimento comunista internazionale. Höbel ha scritto un libro d'“altri tempi”, per la combinazione tra una visione partecipe e un ricco lavoro d'archivio. La lettura critica di Una vita partigiana invita a riflettere non solo sull'esperienza politica e umana di Luigi Longo, ma anche su un doppio livello interpretativo: quello della storia del Pcd'I, dei dirigenti e militanti dei partiti comunisti, e quello degli storici che ne raccontano la storia.

La prospettiva interpretativa di Höbel (fornire prove dell'esemplarità di Longo) non è occultata - del resto il libro è frutto dell'attività dell'autore come borsista presso la Fondazione Luigi Longo - ed è sostenuta da un lavoro accurato e da un'argomentazione che si snoda lungo più di 350 pagine con una riuscitissima selezione della bibliografia ma soprattutto con un uso delle fonti d'archivio organico e approfondito. Vi emergono nuclei tematici di grande importanza, che forniscono ulteriori piste di ricerca. C'è per esempio il tema dei momenti chiave nelle scelte di vita dei militanti comunisti. Per "Gallo" quel momento fu l'esperienza come soldato ${ }^{17}$, poiché le storture della vita militare gli fecero scoprire l'esistenza di un sistema responsabile delle disgrazie umane e che l'ordine gerarchico del mondo inculcato nelle scuole altro non era se non la legittimazione della disuguaglianza. Vi sono correlate varie questioni utili allo studio delle biografie dei militanti: il ruolo del servizio militare nella formazione politica e nelle scelte di vita, il rifiuto delle armi o l'apprendimento militare in vista della lotta

popolazione» (Ibidem, p. 87).

${ }_{15}$ Ibidem, p. 268. Anche poco oltre, a p. 276, l'unità di azione tra socialisti e comunisti nella guerra di Spagna di cui scrive nel 1939 su «Lo Stato operaio» conterebbe in nuce la politica di Longo durante la Resistenza. Scrivendo sempre sulla stessa rivista, nel 1927, Longo avrebbe avuto una «intuizione importante» partendo dalla militarizzazione fascista della gioventù e mettendo in campo la prospettiva insurrezionale contro il fascismo (p. 94). Negli anni della svolta - e in meno di due pagine (pp. 129-130) - "Gallo" avrebbe formulato almeno due previsioni, una lucida e l'altra esatta, sui pericoli della guerra, soprattutto per i lavoratori. A p. 234 Longo, nel 1936, avrebbe formulato la lucida previsione secondo cui l'asse Hitler- Mussolini si sarebbe rinsaldato nonostante i contrasti e avrebbe portato alla guerra mondiale.

${ }^{16}$ Ibidem, p. 316.

${ }_{17}$ Ibidem, pp. 23-24. 
armata, la combinazione tra la condanna della guerra e la necessità di armarsi contro il fascismo. L'autore, inoltre, traccia bene gli spostamenti di Longo tra Francia, Spagna, Svizzera, Russia e altre località di passaggio e quando riferisce dell'arresto a Zurigo, per documenti falsi, accenna alla rete dell'antifascismo europeo che allora (1929) aveva iniziato a costituirsi ${ }^{18}$. È un ottimo punto di partenza per questioni e ricerche sulla costruzione delle reti di sostegno dentro e fuori dall'Italia, sulla combinazione e la sovrapposizione tra istanze politiche e solidarietà umana, sulle continuità e discontinuità esistenti in queste esperienze e sulle eredità che queste hanno lasciato nell'interpretazione dei vari livelli - nazionale, internazionale, transnazionale - di militanza.

Höbel riferisce anche nel dettaglio e con la consueta scrittura partecipe le peregrinazioni di Longo per commissariati, prigioni, campi di internamento e smistamento. L'autore non vittimizza i protagonisti, nemmeno quando questi sono temporaneamente sconfitti; sembra dunque interessante approfondire come si costruiscono e si trasmettono - in sede storiografica, memorialistica, o di dibattito pubblico - le categorie di vittime, carnefici, eroi. Dall'altro lato, le vicende di Longo e del Pcd'I degli anni raccontati da Höbel restituiscono scenari di lotta, repressione e violenza che sarebbero cambiati all'indomani della liberazione. Proprio durante la segreteria di Longo, anni a cui l'autore aveva già dedicato il suo precedente lavoro ${ }^{19}$, alcuni dei dirigenti del PCI - uno dei più duri fu Giorgio Amendola - avrebbero cercato di sopire le istanze di giovani militanti rivendicando al partito il monopolio della lotta e il controllo della violenza. Un'analisi sul lungo periodo dei diversi contesti di lotta e repressione, nonché dell'uso e del significato attribuito alla violenza, possono arricchire le interpretazioni sulla cultura politica del partito e sul dibattito maturato intorno a questi temi all'interno e all'esterno del PCI.

Risulta poi particolarmente interessante il filo rosso sui meccanismi regolatori degli organismi dirigenti del partito. Il dibattito politico di cui Longo fu protagonista, dalla condivisione delle posizioni di Bordiga all'allontanamento dalla sinistra bordighiana ${ }^{20}$, dalla costruzione - insieme a Secchia - della "nuova sinistra" nel gruppo dirigente ${ }^{21}$, sino alla ricerca dell'accordo con Togliatti $^{22}$, sono spunti per l'indagine sul funzionamento della leadership del partito. La storia del Pcd'I di quegli anni e la vicenda di "Gallo" - una vicenda di contrasti che però non arrivò mai alla rottura con

\footnotetext{
${ }^{18}$ Ibidem, p. 126.

${ }^{19}$ HÖBEL, Alexander, Il PCI di Luigi Longo (1964-1969), Napoli, Edizioni Scientifiche Italiane, 2010.

${ }^{20}$ ID., Luigi Longo, cit., p. 63.

${ }^{21}$ Ibidem, p. 85.

${ }^{22}$ Ibidem, p. 122.
} 
"Ercoli" 23 - è un osservatorio sulla tenuta d'insieme degli organismi collettivi e su alcuni concetti chiave per lo studio dei partiti e dei gruppi, come corrente, frazionismo, espulsione, rottura, unità. Sono solo alcuni degli spunti che emergono dal ricchissimo libro di Höbel, e ce ne sarebbero molti altri; la mancata condivisione della scelta interpretativa non può tacere il merito dell'autore di aver raccontato con ricchezza documentale, minuzia e passione argomentativa un'esperienza politica straordinaria del Novecento.

23 Ibidem, p. 131, p. 166. 


\section{* L'autore}

Giulia Strippoli ha conseguito il dottorato in Studi Storici presso l'Università degli Studi di Torino con una tesi sui rapporti tra i partiti comunisti francese, italiano e portoghese e i movimenti studenteschi degli anni sessanta. Dal 2013 svolge attività di ricerca presso l'Istituto di Storia Contemporanea dell'Unversidade Nova de Lisboa. Studia soprattutto temi connessi alla storia dei partiti comunisti, dei movimenti studenteschi, delle organizzazioni della sinistra rivoluzionaria.

URL: < http://www.studistorici.com/progett/autori/\#Strippoli >

\section{Per citare questo articolo:}

STRIPPOLI, Giulia, «Recensione: Alexander HÖBEL, Luigi Longo, una vita partigiana (1900-1945), Roma, Carocci, 2013, 374 pp.», Diacronie. Studi di Storia Contemporanea : Un bilancio della scommessa democratica della Chiesa cattolica, 29/06/2016,

URL:< http://www.studistorici.com/2016/06/29/strippoli_numero_26/ >

\section{Diacronie Studi di Storia Contemporanea 3 www.diacronie.it}

Risorsa digitale indipendente a carattere storiografico. Uscita trimestrale. redazione.diacronie@hotmail.it

Comitato di redazione: Jacopo Bassi - Luca Bufarale - Elisa Grandi - Antonio César Moreno Cantano - Deborah Paci - Fausto Pietrancosta - Alessandro Salvador - Matteo Tomasoni - Luca Zuccolo

Diritti: gli articoli di Diacronie. Studi di Storia Contemporanea sono pubblicati sotto licenza Creative Commons 3.0. Possono essere riprodotti e modificati a patto di indicare eventuali modifiche dei contenuti, di riconoscere la paternità dell'opera e di condividerla allo stesso modo. La citazione di estratti è comunque sempre autorizzata, nei limiti previsti dalla legge. 